\title{
COMPROBACIÓN DEL COMPORTAMIENTO CAÓTICO EN BOLSA DE VALORES DE COLOMBIA
}

\author{
Katherine Julieth Sierra Suárez ${ }^{1}$ \\ Juan Benjamín Duarte Duarte ${ }^{2}$ \\ Juan Manuel Mascareñas Pérez-Iñigo ${ }^{3}$
}

\section{Resumen}

La hipótesis de eficiencia en los mercados bursátiles es uno de los supuestos básicos de los modelos de valoración de activos, tales como el Capital Asset Pricing Model y Arbitrage Pricing Theory, y sostiene que no es posible predecir los precios de un activo financiero, dado que se comportan aleatoriamente. Contrariamente, la hipótesis de mercado fractal afirma que los precios tienen estructura caótica, y podrían ser predichos a partir de modelos no lineales, rechazando así la hipótesis de mercado eficiente e invalidando los supuestos de los modelos valoración de activos. Este trabajo busca evidenciar el comportamiento caótico en el mercado bursátil colombiano con el fin de rechazar o aceptar la hipótesis de mercado eficiente, usando metodologías como: gráficos de precios, gráficos de recurrencia, dimensión de correlación, coeficiente de Hurst, exponente de Lyapunov y el test de Brock, Decher y Scehinkman. Los resultados revelan que los activos muestran indicios de comportamiento caótico para periodos al alza y aleatorio para periodos a la baja, apoyando así la hipótesis de mercado fractal. Estos hallazgos podrían respaldar el uso de modelos no lineales para la predicción de los precios en los periodos al alza y rechazar la eficiencia del mercado bursátil colombiano.

Palabras clave: Teoría de caos, Hipótesis de Mercado Eficiente, Hipótesis de Mercado Fractal, Modelos Autorregresivos. Clasificación JEL: C01, C22, D52, G14

\footnotetext{
${ }^{1}$ E Ingeniera Industrial, Universidad Industrial de Santander-Colombia. Correo electrónico: katjulss@gmail.com

${ }^{2}$ Magister en Finanzas de Empresas, Profesor Asociado Escuela de Estudios industriales y Empresariales, Universidad Industrial de Santander-Colombia. Correo electrónico: jduarte@uis.edu.co

${ }^{3}$ Doctor en Administración de Empresas, Profesor Catedrático Economía Financiera, Universidad Complutense de Madrid-España. Correo electrónico: jmascare@ccee.ucm.es
} 


\section{VERIFICATION OF CHAOTIC BEHAVIOR IN COLOMBIA STOCK MARKET}

\section{Abstract}

The efficient stock market hypothesis is one of the basic assumptions of asset pricing models such as the Capital Asset Pricing Model and Arbitrage Pricing Theory and states that financial asset prices can not be predicted since they behave randomly. Conversely, the fractal market hypothesis states that prices have chaotic structure and could be predicted with nonlinear models, rejecting the efficient market hypothesis and invalidating the assumptions of asset pricing models. This paper seeks to evaluate the chaotic behavior in the Colombian stock market in order to reject or accept the efficient market hypothesis, using tools such as price charts, recurrence plots, correlation dimensión, Hurst coefficient, Lyapunov exponent and BDS test. The results show that the Colombian financial assets reveal chaotical behavior for "bull" market subperiods and randomized for "bear" market subperiods, supporting the fractal market hypothesis. These findings may support the use of nonlinear models for predicting prices in "bull" market episodes and reject the Colombian stock market efficiency.

Keywords: Chaos Theory, Efficient Market Hypothesis, Fractal Market Hypothesis, Autoregressive Models. JEL Classification: C01, C22, D52, G14

\section{Introducción}

Cowles \& Jones (1937), Alexander (1961) y Fama (1965), trabajan sobre la hipótesis de mercados eficientes $(\mathrm{EMH})$, que afirma que un mercado eficiente es una competencia equitativa, en la cual la información está libremente disponible para todos los participantes y se refleja en forma instantánea en los precios de los activos. Asumiendo que el mercado es eficiente, los cambios en los precios solo se producirán cuando se incorporen noticias de forma aleatoria, por lo que no es posible predecir sistemáticamente el comportamiento futuro de los activos.

Sin embargo, algunos supuestos de la EMH no se cumplen en las series empíricas, entre ellos, distribución normal, independencia, homocedasticidad y no autocorrelación. Por esto, Box \& Jenkins (1970); Engle (1982) y Bollerslev (1986) proponen modelos con términos autorregresivos y de medias móviles (ARMA, ARCH y $\mathrm{GARCH}$, respectivamente) que explican el comportamiento del mercado, usando información endógena de tipo histórico, rechazando así la eficiencia del mercado, y dando paso a la búsqueda de modelos de pronósticos como los sugeridos por Jarrett \& Schilling (2008). No obstante, Grau (1996) afirma que estos ajustes lineales no pueden reproducir completamente la dinámica del sistema económico y sólo representan un número limitado de comportamientos. Sin embargo, la teoría del caos propuesta por Lorenz (1963), Takens (1981), Mandelbrot (1961); ha surgido como alternativa para ajustar el comportamiento no lineal y la dependencia de largo plazo en las series temporales. Una buena definición de Sistema Caótico es la presentada por Gálvez (2005): un sistema caótico posee un atractor extraño o ecuación, con características fractales como la autosimilitud, que gobierna el comportamiento determinista (no aleatorio), es sensible a las condiciones iniciales (un pequeño cambio en una condición inicial del sistema, lleva a un gran cambio en el resultado final), es periódico (recurrente) y transitivo (irreducible). 
En los años 80, la teoría del caos es aplicada con resultados importantes en las series económicas y posteriormente en los mercados financieros. En base a esto, Peters (1994) plantea la hipótesis de mercado fractal, que afirma que la información ingresa al mercado según el horizonte temporal de los inversores. Esta hipótesis se caracteriza por ineficiencia, memoria de largo plazo, aleatoriedad local y determinismo global, lo cual permite hacer predicciones en el corto plazo, en consecuencia, diversos autores han buscado indicios de comportamiento caótico en los cambios de los precios financieros, que confirmen la existencia de mercados fractales en lugar de mercados eficientes.

Entre los autores que han investigado el comportamiento caótico en las series financieras se destacan Lipka \& Los (2003), quienes encuentran dependencias de largo plazo en las bolsas europeas aunque resaltan que estas son antipersistentes y se revierten rápidamente a la media sin generar rendimientos significativos; en ese mismo año, Kyaw, Los, \& Zong (2003) estudian los índices bursátiles de Latinoamérica, encontrando persistencia en casi todas las series, excepto en la de Colombia, concluyendo que aunque los largos períodos de calma en los mercados persistentes daban la impresión de predictibilidad, no eran suficiente para obtener rendimientos extraordinarios. Basados en los trabajos anteriores, Espinosa, Parisi, \& Parisi (2007) estudian los mercados americanos (sin incluir a Colombia) usando varías metodologías para evaluar comportamiento no lineal y la dependencia de las condiciones iniciales, concluyendo que todas las series presentan evidencia de no aleatoriedad, lo cual apoya la hipótesis de mercado fractal, justificando la búsqueda de modelos para realizar predicciones. En cuanto al mercado colombiano, Velásquez \& Restrepo (2012), en un estudio similar al de Espinosa et al, encuentran comportamiento caótico en la serie del IGBC en el periodo de 2001 a 2011. Dado estos resultados, en este artículo se usa la me- todología planteada por Espinosa et al, con el fin evaluar las principales acciones de la Bolsa de Valores de Colombia (BVC) y se comparan los resultados con los del índice general de la bolsa como representante del mercado, para cinco subperiodos iguales desde el año 2002 hasta el 2012, evaluando así la evolución de la eficiencia del mercado. El presente documento se divide en cinco capítulos: el primero presenta la introducción; luego se presenta la metodología, los resultados y la discusión; mientras, el último capítulo corresponde a las conclusiones.

\section{Métodos}

La metodología utilizada en el presente trabajo es de tipo empírico-analítico; además, corresponde a un estudio descriptivo, con un enfoque mixto (cuantitativo y cualitativo) para caracterizar el comportamiento de los precios de los activos financieros seleccionados. La población de interés son las principales acciones del mercado bursátil de Colombia, tomando como población de control el Índice General de la Bolsa de Colombia (IGBC); los datos utilizados son las series históricas de los precios de los activos financieros seleccionados, suministradas por la Bolsa de Valores de Colombia.

Con base en la metodología planteada por Espinosa, Parisi, \& Parisi (2007), la búsqueda de caos en el mercado de Colombia se divide en tres partes, la primera consiste en filtrar las series con modelos autorregresivos, la segunda en usar herramientas de comprobación de caos y la última en usar un test estadístico para verificar los resultados de la segunda sección.

En la primera parte se obtienen series de residuos no autocorrelacionados, mediante el ajuste de modelos ARIMA-GARCH, los resultados del test DFA confirmaron la estacionariedad mientras que los test de Bartlett (1946) y Ljung \& Box (1978) no pueden rechazar la autocorrelación, permitiendo así el ajuste de modelos ARIMA, 
mediante la metodología planteada por Box \& Jenkins (1970), para obtener series de residuos estandarizados sin autocorrelación; luego el test ARCH de Engle (1982), evalúa la necesidad de ajustar modelos GARCH si la serie no es homocedastica; finalmente los residuos se evaluan mediante la prueba de Durbin-Watson para determinar si presentan autocorrelación; y se eligen los más adecuados mediante los criterios de información de Akaike, Schwarz y HannanQuinn, los cuales permiten seleccionar el ajuste con el menor número de coeficientes y máxima verosimilitud. Es importante resaltar, que en este trabajo los modelos ARIMA-GARCH se usan como filtros para pruebas posteriores, pero también pueden ser un buen ajuste de la serie a un modelo lineal; que haría inútil la búsqueda de un modelo más complejo.

La segunda parte consiste en usar las herramientas que miden el comportamiento caótico en las series. A nivel cualitativo se analizan las gráficas de precios a diferentes escalas, que deben tener la misma estructura debido a que los inversores negocian en el corto plazo con visión a largo plazo, tal como lo afirma Peters (1994). Por otra parte, el gráfico de recurrencia de Eckmann, Kamphorst, \& Ruelle (1987) representa la integral de correlación definida en (1), preservando la dependencia temporal y espacial en la serie, y se utiliza para mostrar los cambios estructurales y patrones recurrentes e intermitentes en una serie de tiempo; este gráfico es una matriz de distancias representadas con un código de colores, donde el color más claro es la distancia más corta y el color más fuerte es la distancia más grande; así, entre más estructurado sea el gráfico de recurrencia, más determinista es la serie; en forma cuantitativa, la entropía de espacio temporal (STE) es un porcentaje que mide la distribución de estas distancias, indicando aleatoriedad (100), periodicidad (0) o caos (entre 0 y 100).

No obstante, las pruebas cualitativas no son definitivas para determinar que la serie es caótica, por esto se usan las técnicas cuantitativas presentadas a continuación.

La dimensión de correlación se define a partir de la integral de correlación $C^{m}(\varepsilon)$ en la dimensión $m$, la cual es la probabilidad de que dos puntos del atractor estén separados por una distancia menor que la distancia umbral $\varepsilon$. Siendo el número total de observaciones; $x_{i}, x_{j}$ dos observaciones y $H\left(\varepsilon, \bar{x}_{2}, \bar{x}_{j}\right)$ la función de Heaviside con valor cero si $\left(\varepsilon-\left\|\bar{x}_{\imath}-\bar{x}_{j}\right\|\right) \leq 0$ o uno en caso contrario, entonces la integral de correlación se define como:

$C^{m}(\varepsilon)=\lim _{N \rightarrow \infty} \frac{\sum_{i=1}^{N} \sum_{j=1}^{N} H\left(\varepsilon, \bar{x}_{1}, \overline{x_{j}}\right)}{N^{2}}$

Grassberer \& Procacccia (1983) consideran que para valores pequeños de $\varepsilon, C(\varepsilon)=\varepsilon^{D c}$ donde Dc es la dimensión de correlación, entonces $\quad D c=\log C(\varepsilon) / \log \varepsilon$, donde $C(\varepsilon)=\lim _{m \rightarrow \infty} C^{m}(\varepsilon)$. Si la serie es caótica, cuando aumenta el valor de, el valor de tenderá asintóticamente al valor de la dimensión del atractor. Pero si el fenómeno es aleatorio, la dimensión de correlación aumenta indefinidamente con $m$. En sistemas no aleatorios $D c$ se mantendrá siempre por debajo de la dimensión de inmersión y como criterio de decisión si su valor es igual a uno la serie es periódica, si es igual a dos es cuasi-periódica, pero si es mayor de dos el comportamiento es caótico y la dimensión de correlación determina la dimensión del atractor extraño.

El coeficiente de Hurst $(\mathrm{H})$ mide el nivel de persistencia y detecta memoria de largo plazo en las series temporales. Este coeficiente es calculado a través de la ley de potencia $R / S(\tau)=(a * \tau)^{H}$, donde, $a$ es una constante, $\tau$ es el número de observaciones, $H$ es el coeficiente de Hurst, y $R / S(\tau)$ es el estadístico que depende del tamaño de la serie y se define como el rango de variación de la serie sobre su desviación típica, en este trabajo se calcula 
el coeficiente usando el código Matlab de Pérez (2008). Como criterio de decisión, si H>0.5 la serie es persistente, lo cual es indicio de que las tendencias se mantienen, si $\mathrm{H}<0.5$ la serie es antipersistente, pero $\mathrm{si} \mathrm{H}=0.5$ la serie es ruido blanco.

El exponente de Lyapunov mide la separación de dos orbitas cercanas respecto al tiempo y detecta la sensibilidad a las condiciones iniciales en las series. Indica que dos trayectorias dentro del atractor, con separación inicial $\Delta x_{0}$ , divergen en el instante thasta $\Delta x_{t}$; si $t$ tiende a infinito y $\Delta x_{0}$ es pequeño, estas distancias se relacionan mediante la expresión $\delta x_{t} \approx e^{\lambda t} \delta x_{0}$ . Si D es la distancia entre dos orbitas cercanas y $\lambda$ es el exponente de Lyapunov, entonces $D=e^{\lambda t}$. Si la serie converge $\lambda<0$, si es periódica y no converge ni diverge $\lambda=0$, pero si es caotica $\lambda>0$.

Para corroborar los resultados obtenidos anteriormente, en la tercera parte se usa el test estadístico BDS de Brock, Decher, \& Scehinkman (1987) que prueba si una serie es independiente e idénticamente distribuida (i.i.d), o si por el contrario, presenta dependencia lineal, no lineal o comportamiento caótico. Para su cálculo se emplea la integral definida en (1), de forma que si la hipótesis es cierta, se cumplirá la igualdad:

$c^{m}(\varepsilon)=\left[c^{1}(\varepsilon)\right]^{m}$

De hecho, Brock, Decher, \& Scehinkman (1987) demostraron que si la varianza es $\sigma_{m}{ }^{2}(\varepsilon)$ y de- pende de la integral de correlación de dimensión 1 y de $\boldsymbol{K}(\boldsymbol{\varepsilon})$ que es la probabilidad de que tres puntos estén a una distancia menor de $\boldsymbol{\varepsilon}$. Entonces el estadístico (3) permite contrastar la hipótesis nula (2).

$$
\frac{\sqrt{N}\left[c^{m}(\varepsilon)-\left[c^{1}(\varepsilon)\right]^{m}\right]}{\sigma_{m}(\varepsilon)} \rightarrow N(\mathbf{0}, \mathbf{1})
$$

\section{Resultados}

A continuación, se presentan los datos utilizados en el presente estudio con la estimación de los estadísticos básicos, además de las pruebas y modelos descritos en la metodología.

\section{Datos y Estadísticas Básicas.}

Los datos seleccionados corresponden a los precios de cierre de los principales activos que representan aproximadamente el $60 \%$ de la composición del índice, además del índice general de la bolsa como representación del mercado. Se omiten los días en los que la Bolsa no operó y los que presentan valores nulos. Las series se analizan en el periodo total, y en subperiodos iguales de 525 (índice) y 510 (demás activos) datos, con el fin de observar la evolución de la eficiencia del mercado bursátil a través de los años. Se toma como variable, la rentabilidad continua de cada activo porque los modelos autorregresivos se construyen con series estacionarias, tomando los retornos que estén en el intervalo \pm 3.5 veces la desviación típica de las series (Gimeno, 2000). 
Tabla 1. Activos Financieros Seleccionados

\begin{tabular}{|c|c|c|c|c|}
\hline Activo & Nemotūcnico & $\mathbf{N}$ & Fecha Inicial & Fecha Final \\
\hline $\begin{array}{l}\text { Índice General Bolsa de } \\
\text { Colombia }\end{array}$ & IGBC & 2625 & 03/08/2001 & $15 / 06 / 2012$ \\
\hline Ecopetrol & Ecopetrol & 1020 & $21 / 04 / 2008$ & 03/07/2012 \\
\hline $\begin{array}{l}\text { Pacific Rubiales Energy } \\
\text { Corporation }\end{array}$ & PREC & 510 & 08/06/2010 & 03/07/2012 \\
\hline Preferencial Bancolombia & PFBCOLOM & 2040 & $27 / 01 / 2004$ & 03/07/2012 \\
\hline $\begin{array}{l}\text { Grupo Inversiones } \\
\text { Suramericana }\end{array}$ & Gruposura & 510 & $27 / 05 / 2010$ & 29/06/2012 \\
\hline Cementos Argos & Cemargos & 1530 & 06/03/2006 & 29/06/2012 \\
\hline Isagen & Isagen & 1020 & $16 / 04 / 2008$ & 03/07/2012 \\
\hline Bancolombia & BColombia & 2550 & $31 / 10 / 2001$ & 03/07/2012 \\
\hline
\end{tabular}

Fuente: Elaboración Propia.

Al estimar las estadísticas básicas para los diferentes activos se encuentra que: todas las series presentan medias positivas estadísticamente significativas en los años 2001 a 2006; el IGBC muestra asimetría negativa durante todo el periodo y significativa hasta el año 2006 a pesar de que los valores extremos han sido eliminados; las series son leptocúrticas y la significancia del estadístico Jarque-Bera rechaza la hipótesis de distribución Normal en los retornos, lo cual según Espinosa (2008), es indicio de que las series han sido generadas por procesos no lineales. Por otro lado la prueba de Dickey y Fuller Aumentada (DFA) rechaza la existencia de raíz unitaria en las series de retornos; mientras que el intervalo de Bartlett y el estadístico de Ljung \& Box (1978) muestran que las series presentan autocorrelación, principalmente en el primer rezago y en el subperiodo de 2008 a 2010.

\section{Filtros ARIMA-GARCH}

En las series que no se identificaron rezagos autocorrelacionados, no es necesario ajustar modelos ARIMA; para las demás series se usa la metodología planteada por Box-Jenkins. Para cada modelo seleccionado se evalúa nuevamente la autocorrelación en los residuos usan- do el test de Bartlett y el test LB, la cual no es significativa en los diez primeros rezagos. También se verifica la homocedasticidad mediante el test $\mathrm{ARCH}$ (para las series que no se encontró un modelo ARIMA, se hace una regresión respecto a la media y se analizan los residuos resultantes), el cual rechaza la hipótesis nula de que las series son homocedasticas; es importante resaltar que en el primer subperiodo de las acciones (ordinaria y preferencial) de Bancolombia y segundo de Isagen, no se rechaza la hipótesis de homocedasticidad, no obstante al observar el correlograma de residuos al cuadrado se identifican autocorrelaciones en la varianza, por lo tanto se ajustan modelos GARCH para cada activo, siguiendo la metodología ARIMA en los residuos al cuadrado. La tabla 2, muestra un resumen de los modelos ajustados para cada activo, los cuales permiten generar una serie de residuos sin autocorrelaciones significativas en sus primeros diez rezagos, que pudieran afectar las pruebas de comportamiento caótico; las series de residuos representaran al sistema original, de tal forma que si éste fuera aleatorio, los residuos seguirían un proceso ruido blanco, pero si fuera caótico, los residuos mostrarían dependencias no lineales, sensibilidad a las condiciones iniciales y memoria de largo plazo. 
Tabla 2. Modelos GARCH()



Notas: Los modelos GARCH incluyen todos los términos y anteriores al del ajuste presentado.

Fuente: Elaboración Propia. 


\section{Herramientas para la comprobación de la teoría del Caos}

Gráfica Precios vs Tiempo a diferentes escalas

Para cada acción, se grafican el periodo total, un periodo de aproximadamente la mitad de los

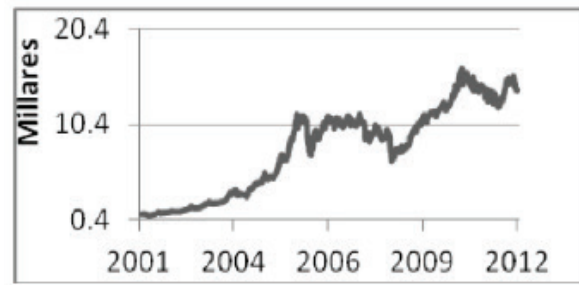

a

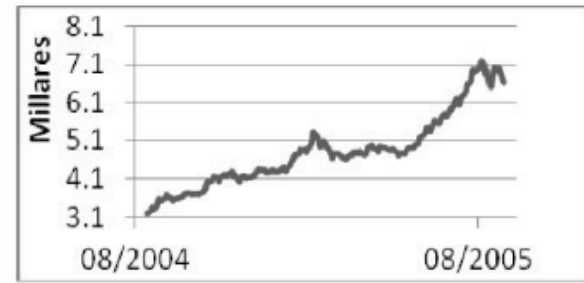

d datos, y de forma sucesiva, periodos más pequeños contenidos en el inmediatamente anterior, de tal forma que al contrastar el comportamiento de los precios en el periodo total con el de periodos de $1.000,500,250, \ldots$, datos diarios, se posible determinar si el comportamiento de la serie es fractal.

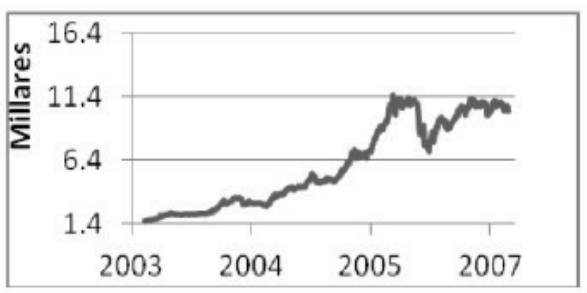

b

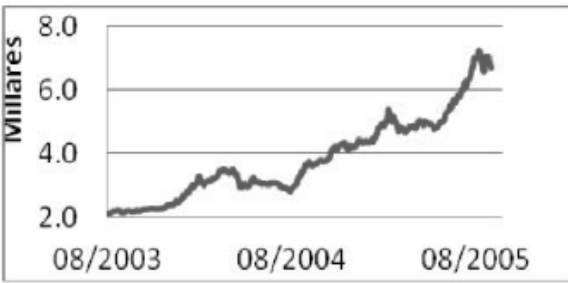

C

Figura 1. Precios del Índice General de la Bolsa, con diferente escala temporal.

Fuente: Elaboración propia

En la Figura 1, se observa que para la serie del IGBC no se pueden distinguir gráficamente los periodos seleccionados, cuando estos sucede la serie graficada es un fractal, lo cual es indicio de que el mercado puede comportarse como un sistema caótico. Este mismo comportamiento se observa en los demás activos, sin embargo, hace falta más análisis antes de concluir que el mercado es fractal.

\section{Gráficos de recurrencia}

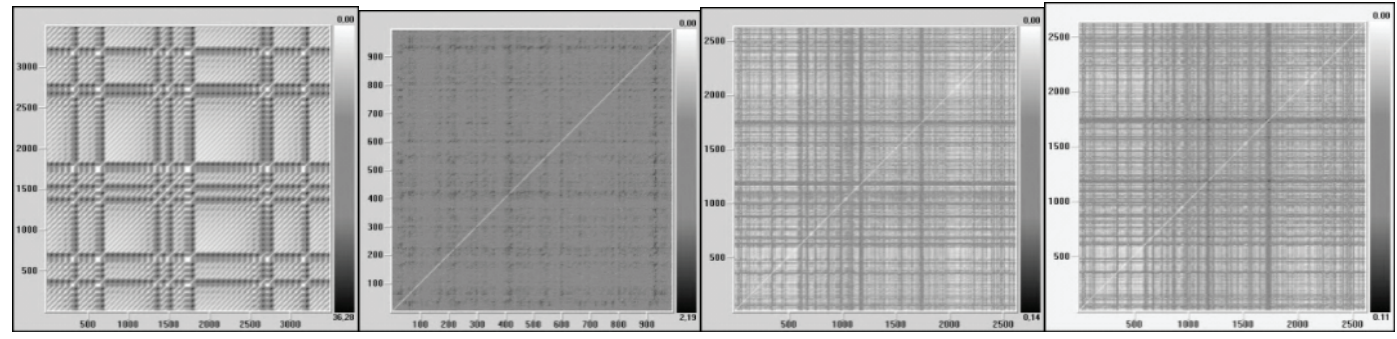

Figura 2. Gráficos de Recurrencia.

Fuente: Elaboración propia usando el software VRA, el cual proporciona también los datos del retardo temporal y la dimensión de inmersión. 
En la Figura 2.a se muestra una serie teórica generada por el atractor de Lorenz y la Figura 2.b representa un proceso de ruido blanco, como se observa en ambas figuras, cuando una serie tiene un comportamiento caótico el gráfico de recurrencia es más estructurado que el de una serie aleatoria. Los gráficos de la serie del IGBC para las rentabilidades (Figura 2.c) y los residuos ARIMA-GARCH (Figura 2.d) muestran que las estructuras marcadas en las rentabilidades se hacen más evidentes en la serie de residuos, esto indica que la serie tiene patrones recurrentes e intermitentes propios de las series deterministas. También se estudia la evolución de los gráficos de recurrencia a través de los subperiodos, encontrándose que los gráficos que corresponden a datos tomados entre finales del 2005 y mediados de 2012 son más uniformes, evidenciando mejora de la aleatoriedad, como también sucede en Ecopetrol e Isagen. Mientras que los gráficos de PREC, Cemargos y Gruposura muestran comportamiento aleatorio en todo el periodo de estudio. Por otro lado, el periodo total y los subperiodos dos, cuatro, y cinco de PFBCOLOM, muestran estructuras recurrentes. Finalmente, BColombia, muestra recurrencia en los dos primeros subperiodos, aleatorio en los subperiodos 3 y 4 , y vuelve a ser caótico en el último periodo.

Para evaluar matemáticamente los gráficos de recurrencia, se usa el porcentaje STE que muestra que la entropía en el IGBC aumenta a medida que el tiempo avanza y genera en promedio un valor de $77 \%$, lo cual es indicio de comportamiento caótico. Sin embargo, al calcular el índice para una serie aleatoria débil, generada por la función «aleatorio» de Excel, se obtiene un STE igual $76 \%$ y para una serie caótica generada por un atractor de Lorenz se obtiene un valor de $57 \%$; entonces se analiza en forma conjunta el gráfico de recurrencia y el porcentaje STE, de tal forma que si el gráfico es caótico, se considera caótico, un porcentaje entre 0 y 100 , pero si el gráfico es aleatorio y el porcentaje STE es mayor a $76 \%$, se considera aleatorio. Para las acciones se observa que la entropía de espacio temporal es en promedio $77 \%$, y el valor máximo (90\%) lo alcanza la serie de Cemargos. En consecuencia, se consideran aleatorios: el último subperiodo de Ecopetrol $(80 \%)$ e Isagen $(78 \%)$, el primer subperiodo de PFBCOLOM (79\%), el periodo total (86\%) y el último (81\%) subperiodo de Cemargos, los subperiodos 3 y 4 de BColombia (77\%), la serie de PREC (79\%) y la de Gruposura (78\%).

\section{Dimensión de Correlación}

El valor de la dimensión de correlación para los periodos total y primero del IGBC, se hace estable en un valor menor a la dimensión de inmersión y mayor a dos, lo cual es un claro indicio de comportamiento caótico; en el siguiente subperiodo parece estabilizarse cerca de 6 , pero luego disminuye y aumenta drásticamente, lo cual hace que el resultado no sea concluyente; entre finales de 2005 y mediados del 2010, la dimensión de correlación muestra aleatoriedad; sin embargo, en el último periodo (2010-2012) hay un claro indicio de caos. En cuanto a las acciones, los resultados son diversos: en la serie de Ecopetrol el resultado del periodo total no es concluyente, pero en los subperiodos se observa una transición de comportamiento caótico a aleatorio; en el periodo total de PFBCOLOM, la tampoco es concluyente, pero al observar los subperiodos los resultados son similares a los encontrados con el grafico de recurrencia para estas series; la dimensión de correlación en la serie Gruposura indica comportamiento caótico; por el contrario, para la serie de Isagen muestra comportamiento aleatorio; por su parte, la serie de Cemargos pasa de ser caótica a aleatoria; finalmente en los subperiodos uno, dos y cuatro de BColombia se observa comportamiento caótico.

\section{Coeficiente de Hurst}

Para medir el nivel de persistencia en las series estudiadas, se calcula el coeficiente de Hurst. En la serie del IGBC, todos los periodos 
muestran que el activo es persistente, aunque esta característica disminuye a través de los años; lo que indica que la serie tiene un comportamiento caótico que disminuye lentamente a través del tiempo. Las demás series producen coeficientes de Hurst mayores a 0.5 , es decir son persistentes, y posiblemente caóticas, excepto las series de Cemargos y Bancolombia, las cuales en sus periodos completos son antipersistentes, es decir se revierten rápidamente a la media y en este sentido son eficientes.

\section{Máximo Exponente de Lyapunov:}

Para el IGBC, se observa que la sensibilidad a las condiciones iniciales es totalmente clara en el periodo completo y los subperiodos 2, 3 y 5 , mientras que en el primer subperiodo el exponente es cero, esto indica que la serie no converge ni diverge, además, en el periodo cuatro el valor del exponente de Lyapunov es tan pequeño que podría indicar un comportamiento similar. Por otro lado en los otros activos los exponentes son positivos, indicando presencia de sensibilidad a las condiciones iniciales, aunque hay indicios de periodicidad en: el primer subperiodo de Ecopetrol, las series de PREC y Gruposura, todos los subperiodos (excepto 2 y 3 ) de PFBCOLOM, el primer y último subperiodo de Cemargos, el penúltimo subperiodo de Isagen, el periodo total y subperiodo dos de Bancolombia.

\section{Herramienta estadística}

Luego de encontrar indicios de comportamiento caótico en todas las series, se usa el test BDS, para evaluar la independencia, tanto en las series de rentabilidad como en las de residuos, encontrando rechazo a la hipótesis nula de que las series se distribuyen de forma independiente; por tanto el activo podría tener dependencia no lineal o comportamiento caótico, ya que los filtros ARIMA-GARCH eliminan en gran parte las dependencias lineales, Sin embargo en Gruposura el p_valor para todas las dimensiones es igual o mayor al $5 \%$, indicando tendencia aleatoria.

\section{Resumen de resultados}

La tabla 3 muestra los resultados para cada periodo de cada activo. Donde «+» indica comportamiento caótico; «-» comportamiento aleatorio, $y$ «NC» no concluyente.

Los criterios de decisión son los mencionados en la metodología, además de que si el gráfico de recurrencia muestra aleatoriedad y el STE es mayor a $76 \%$, el porcentaje STE indica aleatoriedad. En el resumen se estima una serie como caótica cuando hay cuatro pruebas con hallazgo positivo de comportamiento caótico, de lo contrario se considera aleatoria. 
Tabla 3. Resultados test de comprobación de caos en los activos financieros.

\begin{tabular}{|c|c|c|c|c|c|c|c|c|}
\hline Activo & Periodo & $\begin{array}{l}\text { Gr6f. } \\
\text { Rec. }\end{array}$ & $\begin{array}{l}\text { STE } \\
(\%)\end{array}$ & $\begin{array}{l}\text { Dim. } \\
\text { Gorr. }\end{array}$ & Hurst & Lyapunov & BDS & Resumen \\
\hline \multirow{6}{*}{ IGBC } & Total & + & + & + & + & + & + & Caótico \\
\hline & $\mathrm{P} 1$ & + & + & + & + & $\mathrm{NC}$ & + & Caótico \\
\hline & P2 & + & + & $\mathrm{NC}$ & + & + & + & Caótico \\
\hline & P3 & - & - & - & + & + & + & Aleatorio \\
\hline & P4 & - & - & - & + & $\mathrm{NC}$ & + & Aleatorio \\
\hline & P5 & - & - & + & + & NC & + & Caótico \\
\hline \multirow{3}{*}{ Ecopetrol } & Total & + & + & NC & + & + & + & Caótico \\
\hline & $\mathrm{P} 1$ & + & + & + & + & NC & + & Caótico \\
\hline & $\mathrm{P} 2$ & - & - & - & + & + & + & Aleatorio \\
\hline PREC & Total & - & - & $\mathrm{NC}$ & + & NC & + & Aleatorio \\
\hline \multirow{5}{*}{ PFBColom } & Total & + & + & NC & + & NC & + & Caótico \\
\hline & P1 & - & - & - & + & NC & + & Aleatorio \\
\hline & $\mathrm{P} 2$ & + & + & - & + & + & + & Caótico \\
\hline & P3 & - & + & - & + & + & + & Caótico \\
\hline & $\mathrm{P} 4$ & + & + & + & + & $\mathrm{NC}$ & - & Aleatorio \\
\hline Gruposura & Total & - & - & + & + & NC & - & Aleatorio \\
\hline \multirow{4}{*}{ Cemargos } & Total & - & - & + & - & + & + & Caótico \\
\hline & $\mathrm{P} 1$ & - & + & + & + & $\mathrm{NC}$ & + & Caótico \\
\hline & $\mathrm{P} 2$ & - & + & + & + & + & + & Caótico \\
\hline & P3 & - & - & - & + & NC & + & Aleatorio \\
\hline \multirow{3}{*}{ Isagen } & Total & + & + & - & + & NC & + & Aleatorio \\
\hline & $\mathrm{P} 1$ & + & + & - & + & $\mathrm{NC}$ & + & Caótico \\
\hline & P2 & - & - & NC & + & + & + & Aleatorio \\
\hline \multirow{6}{*}{ Bcolombia } & Total & + & + & NC & - & NC & + & Caótico \\
\hline & $\mathrm{P} 1$ & + & + & + & + & + & + & Caótico \\
\hline & $\mathrm{P} 2$ & + & + & + & + & NC & + & Caótico \\
\hline & P3 & - & - & $\mathrm{NC}$ & + & + & + & Aleatorio \\
\hline & $\mathrm{P} 4$ & - & - & + & + & + & + & Caótico \\
\hline & P5 & + & + & NC & + & + & + & Caótico \\
\hline
\end{tabular}

Fuente: Elaboración Propia, basado en el gráfico presentado como resumen por Espinosa et al (2007).

En la tabla 3 y la Figura 3 se observa que el mercado financiero muestra indicios de comportamiento caótico en los periodos entre 2001 y 2006, entre 2006 y 2008 no hay un patrón definido, pero entre 2008 y 2010, se observa nuevamente comportamiento caótico y entre 2010 y 2012 el mercado tiende a ser aleatorio. 


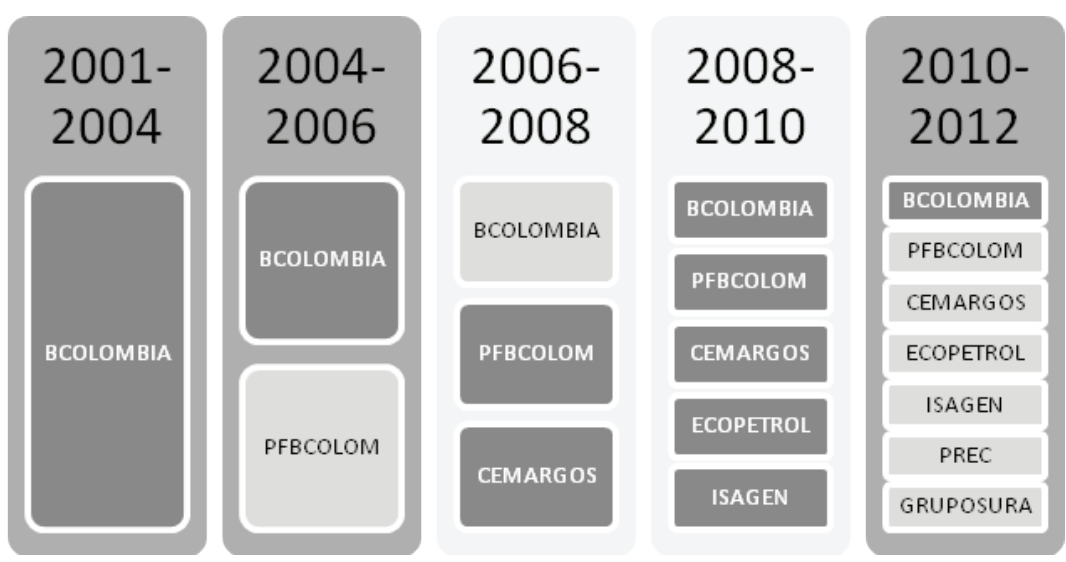

Figura 3. Resultados por subperiodos

Notas: Los colores claros corresponden a series aleatorias mientras que los colores oscuros corresponden a series caóticas; como serie representativa del mercado, los cuadros de fondo representan el comportamiento del IGBC en cada periodo. Fuente: Elaboración Propia.

\section{Discusión de resultados}

Peters (1994), en su hipótesis de mercado fractal, afirma que en periodos de crisis, los inversores de largo plazo prefieren salir del mercado o disminuir su horizonte de inversión, produciendo cambios bruscos en la dinámica del mercado y reduciendo la estabilidad para los inversores de corto plazo. En la Figura 4 se sintetizan los resultados de la sección anterior, observándose que los periodos con tendencia predominante al alza (P1, P2, P4) se comportan de forma caótica, es decir los precios tienen una estructura que los puede hacer predecibles, por el contrario, en el periodo con fuerte tendencia a la baja (P5) los activos presentan un comportamiento aleatorio que no permite la anticipación de sus precios futuros; mientras que para el periodo sin tendencia marcada (P3) no es posible asociarlo a comportamiento aleatorio o caótico. Este es un hallazgo importante, pues podría indicar que el comportamiento de los precios de los principales activos del mercado bursátil de Colombia son previsibles al menos en el corto plazo, durante periodos con tendencia marcada al alza, tal como lo expone la hipótesis de mercado fractal, justificando la búsqueda de modelos de predicción no lineales por parte de inversionistas y analistas del mercado que deseen especular.



Figura 4. Resultados del mercado en contraste con las tendencias Fuente: Elaboración Propia. 


\section{Conclusiones}

Los periodos que muestran indicio de comportamiento caótico, coinciden con los periodos con tendencia al alza, mientras que el aleatorio coincide con el periodo con tendencia a la baja. Esto podría explicarse por la hipótesis de mercado fractal de Peters (1994), la cual postula que los inversores negocian con horizontes de largo plazo cuando el mercado es rentable, produciendo un equilibrio que hace posible realizar predicciones durante este periodo; pero cuando el mercado cae, los horizontes de inversión cambian y se producen cambios bruscos en la dinámica del mercado, que lo hacen más aleatorio y por tanto eficiente, disminuyendo la posibilidad de realizar predicciones acertadas. Sin embargo, se debe tener en cuenta que no se tienen suficientes periodos al alza y a la baja, para corroborar con mayor certeza este comportamiento. No obstante, el periodo mixto evidencia que para obtener resultados concluyentes, los estudios que buscan caracterizar el comportamiento de series financieras, deben tomar en cuenta las tendencias del mercado a pesar de que se esté trabajando con rentabilidades, las cuales son consideradas datos sin estacionalidad.

En los periodos en que los activos inician operaciones en el mercado, la mayoría de ellos muestran comportamientos caóticos, esto podría estar relacionado con la baja frecuencia de negociaciones. Por lo tanto, sería interesante indagar sobre este posible fenómeno, que podría dar lugar a ganancias extraordinarias por parte de un inversor al predecir el comportamiento de activos que ingresen por primera vez al mercado bursátil.

Por otra parte, se observa que el comportamiento del índice general, no necesariamente refleja el comportamiento de los principales activos; lo que es más, en algunos casos el índice muestra comportamiento opuesto al de las acciones individuales. En consecuencia, se deben tomar con precaución las conclusiones de estudios que busquen caracterizar un mercado bursátil evaluando solamente el comportamiento del principal índice bursátil del mercado.

En general, se comprueba mediante el presente trabajo que el mercado bursátil colombiano pasó de ser caótico a aleatorio en el tiempo, lo cual podría dar indicios de mejoramiento de la eficiencia del mercado en Colombia.

Finalmente, se debe tener en cuenta que las pruebas de caos requieren un mínimo de 500 datos por periodo o subperiodo de estudio, lo cual supone una limitación al analizar los activos nuevos y al dividir los periodos totales según la tendencia del mercado.

\section{Referencias}

Alexander, S. (1961). Price Movements in Speculative Markets: Trends or Random Walks. Industrial Management Review, 2: 7-26.

Bartlett, M. S. (1946). On the Theoretical Specification of Sampling Properties of Autocorrelated Time series. Journal of the Royal Statistical Society, 27: 27-41.

Bollerslev, T. (1986). Generalized Autoregressive Conditional Heteroskedasticity. Journal of Econometrics, 31: 307-327.

Box, G., \& Jenkins, G. (1970). Time Series Analysis: Forecasting and Control. San Francisco: Holden Day.

Brock, W. A., Decher, W., \& Scehinkman, J. (1987). A test for independence based on the correlation dimension. Madison: University of Winconsin at Madinson.

Cowles, A., \& Jones, H. (1937). Some A Posteriori Probabilities in Stock Market Action. Econometrica, 5: 208-294.

Eckmann, J. P., Kamphorst, S. O., \& Ruelle, D. (1987). Recurrence Plots of Dynamical Systems. Europhysics Letters, 4(9): 973-977.

Engle, R. F. (1982). Autoregressive Conditional Heterocedasticity with Estimates of the Variance of United Kingdom Inflation. Econometrica, 50: 987-1008.

Espinosa, C. (2008). Comportamiento caótico en los mercados búrsatiles latinoamericanos. Análisis Económico. 
Espinosa, C., Parisi, F., \& Parisi, A. (2007). Evidencia de comportamiento caótico en indices bursátiles americanos. El Trimestre Económico, 901-927.

Fama, E. F. (1965). Efficient capital market: a review of theory and empirical work. Journal of Finance, 24: 383-417.

Gálvez, E. T. (2005). Análisis Fractal del Mercado de Valores de México (1978-2004). México: Instituto Politécnico Nacional.

Gimeno, R. (2000). Análisis Caótico de Series Temporales Financieras de Alta Frecuencia. El Contrato de Futuro sobre el Bono Nocional a 10 años. Madrid: Universidad de Comillas, Tesis Doctoral.

Grassberer, P., \& Procacccia, I. (1983). Characterization of Strange Attractors. Physical Review Letters, 50(3): 346.

Grau, P. (1996). Economía Dinámica Caótica: Una aplicación al mercado de capitales español. Madrid: Universidad Complutense de Madrid.

Jarrett, J. E., \& Schilling, J. (2008). Daily variation and predicting stock market returns for the frankfurter börse. Jour nal of Business Economics and Management, 189-198.

Kyaw, N. A., Los, C. A., \& Zong, S. (2003). Persistence Characteristics of Latin American Financial Markets. Kent: Kent State University.
Lipka, J. M., \& Los, C. A. (2003). Long-Term Dependence Characteristics of European Stock Indices. Kent: Kent State University.

Ljung, G. M., \& Box, G. E. (1978). On a measure of lack of fit in time series models. Biometrika, 297-303.

Lorenz, E. N. (1963). Deterministic nonperiodic Flow. Journal of Atmospheric Sciences, 20: 130-141.

Mandelbrot, B. (1961). Stable Paretian Random Functions and the Multiplicative Variation of Income. Econométrica, 517-543.

Pérez, R. (2008). Programa en Matlab para el cálculo del exponente de Hurst y dimensión fractal de series de tiempo. Bol-e: boletín electrónico del Centro de Geociencias, 4(3). Disponible en: http://www.geociencias. unam.mx/ bole/eboletin/progHurstyD1108.pdf

Peters, E. E. (1994). Fractal Market Analysis. Denver: Wiley Finance Editions.

Takens, F. (1981). Detecting Strange Attractors in Turbulence. Lecture Notes in Mathematics, 366-381.

Velásquez, H., \& Restrepo, J. H. (2012). Análisis del índice general de la bolsa de valores de Colombia y sus rendimientos desde la teoría del caos, 2001-2011. Semestre económico, 79-98. 